\title{
An Optimum Structure of Scalable Capacitors in 3D Crosspoint Memory Technology
}

\author{
Yuya Tone ${ }^{1,2}$ and Toru Tanzawa ${ }^{1, *}$ \\ 1 Faculty of Engineering, Shizuoka University, Hamamatsu 432-8561, Japan; tone.yuya.17@shizuoka.ac.jp \\ 2 Suzuki Corp., Hamamatsu 432-8611, Japan \\ * Correspondence: toru.tanzawa@shizuoka.ac.jp
}

Citation: Tone, Y.; Tanzawa, T. An Optimum Structure of Scalable

Capacitors in 3D Crosspoint Memory Technology. Electronics 2021, 10, 2755. https://doi.org/10.3390/

electronics10222755

Academic Editors: Laurent Artola and Ricardo Reis

Received: 19 October 2021

Accepted: 9 November 2021

Published: 11 November 2021

Publisher's Note: MDPI stays neutral with regard to jurisdictional claims in published maps and institutional affiliations.

Copyright: (c) 2021 by the authors. Licensee MDPI, Basel, Switzerland. This article is an open access article distributed under the terms and conditions of the Creative Commons Attribution (CC BY) license (https:/ / creativecommons.org/licenses/by/ $4.0 /)$.

\begin{abstract}
Memory chips need large capacitors in their periphery to drive boosted word-lines and bit-lines for read and write operations. In a previous work, scalable capacitors were proposed for 3D crosspoint memory to keep the area for the capacitors constant over technology generations. This paper proposes the capacitance models of three types of wiring capacitors: (1) vertical capacitor, (2) vertical and horizontal capacitor with next-neighbor wires connected with the other terminal, and (3) vertical and horizontal capacitor with next-neighbor pairs connected with the other terminal. These models are based on Wong's crossover capacitor model to determine the capacitor structure with the highest capacitance density in 3D crosspoint memory technology. One can determine the best structure through optimizing the process parameters such as the height $H$ of the insulation material between the metal wires and the thickness $T$ of the metal wires and the design rules such as the width $W$ and space $S$ of metal wires. The model accuracy was in good agreement with the measurement of twelve types of capacitor structures fabricated in a $180 \mathrm{~nm} 6$ metal standard CMOS process with the maximum error of $20 \%$. Contour plots of the capacitance density across $H$ vs. $S$ where it is assumed that $W=T=S$ are shown. As a result, the boundary condition regarding $H$ and $S$ is determined per 3D crosspoint memory technology with three, four, or five levels of wires.
\end{abstract}

Keywords: scalable capacitor; capacitor model; 3D crosspoint memory; optimum structure; decoupling capacitor; metal-on-metal

\section{Introduction}

Three-dimensional (3D) crosspoint memory has been developed to fill the performance gap between main memory and NAND Flash for improving the performance of the memory system with the minimum additional cost [1-8]. Memory elements are typically composed of phase change material [1-3] or conductive metal-oxide material [4-8]. To have a sufficient On-Off ratio between a selected memory and deselected ones even with two terminal devices, each memory cell has a selector connected in series with a non-volatile memory element vertically between a word-line (WL) and a bit-line (BL).

Figure 1a illustrates a 3D crosspoint memory array. In this example, the array has two levels of word-lines (WLs) and one level of bit-lines (BLs). There is a non-volatile memory cell at the crosspoint across a WL and a BL. Each cell has non-volatile material and a selecting device, which is shown by a diode in Figure 1a. To increase the bit density, the number of levels of WLs and BLs increases. When the number of accessible memory cells increases for higher performance in terms of read and write bandwidth as the number of levels of WLs and BLs increases, charge pump circuits driving WLs need to have increased drive current every time when the technology is advanced. The area of the charge pump circuits is basically proportional to that of the capacitors required for the demanded drive current. As a result, the control circuit placed in periphery tends to increase as the technology is advanced. 


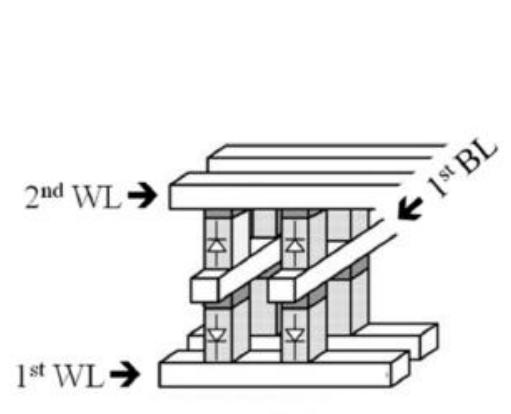

(a)

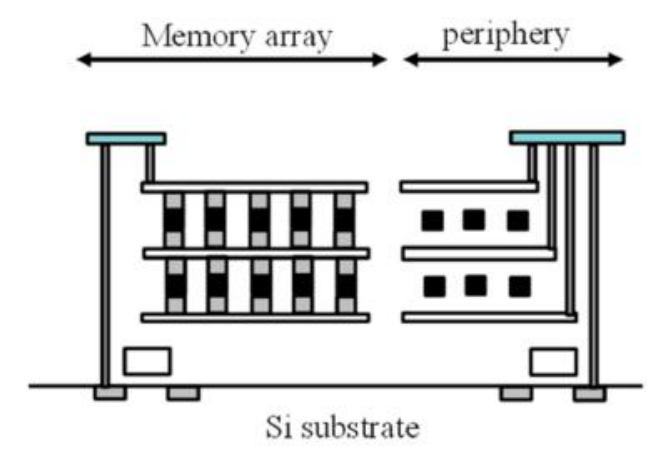

(b)

Figure 1. A structure of the crosspoint non-volatile memory array (a) and cross-sectional view of the chip where CMOS is fabricated under the array $(\mathbf{b})$.

How has that issue been resolved in other memories? In [9-12], one can find circuit designs using memory structures as dense capacitors. Trench capacitors in DRAM have very high dense capacitance. The area of switched capacitor DC-DC voltage converters and decoupling capacitors can be reduced significantly by using trench capacitors $[9,10]$. Similarly, in [11], a highly efficient DC-DC converter using ferroelectric capacitors is developed. For 3D NAND Flash memory, charge pump circuits using tier capacitors that are composed of multiple WLs are developed in [12]. Even in logic chip, charge pumps are sometimes required to generate higher voltages on-chip for power gating. In [13], a voltage doubler was developed using wiring capacitance together with gate capacitance to minimize the capacitor area.

Likewise, to keep the circuit area constant over 3D crosspoint memory technology, a scalable capacitor structure was proposed in [14], as shown in Figure 1b. By using the wires for multiple WLs and BLs as wiring capacitors, the capacitance density is expected to increase in proportion to the number of wires in memory array. Thus, the charge pump area can be constant with scalable capacitors beyond technology transition. Then, what is the best capacitor structure to have the highest capacitance density? The answer would be dependent on the process parameters such as the height of the insulation material between the metal wires and the thickness of the metal wires and the design rules such as the width and space of the metal wires. We have capacitor models for parallel wires [15-17] and for crossover wires [18] at this point. In [15], an effective computation program was developed to obtain the capacitance of wires. In [16], wiring capacitance extraction was presented for post layout simulation with SPICE. In [17], explicit formulas were presented in terms of wire width, wire thickness, dielectric film thickness, and inter-wire spacing for covering arbitrary layouts. In [18], crossover capacitance was focused to have an explicit model for VLSIs with multilevel metal interconnect of arbitrary dielectric and wire thickness, width, and spacing in all layers. Various capacitor structures including both wires and vias are compared in terms of capacitance density, quality factor, and resonant frequency in [19]. However, one cannot determine the best structure for the scalable capacitors in 3D crosspoint memory technology based on those previous studies. Therefore, it should be beneficial for circuit designers to determine it with a simple capacitor model.

In this paper, the capacitance models of three types of wiring capacitors-(1) vertical capacitor, (2) vertical and horizontal capacitor with next-neighbor wires connected with the other terminal, and (3) vertical and horizontal capacitor with next-neighbor pairs connected with the other terminal-are developed based on Wong's crossover capacitor model [18] to determine the capacitor structure with the highest capacitance density in 3D crosspoint memory technology. One can determine the best structure through optimizing the process parameters such as the height $H$ of the insulation material between the metal wires and the thickness $T$ of the metal wires and the design rules such as the width $W$ and space $S$ of metal wires. Section II discusses modeling of the capacitance of scalable capacitors. The model accuracy was in good agreement with the measurement of twelve types of capacitor 
structures fabricated in a $180 \mathrm{~nm} 6$ metal standard CMOS process with the maximum error of $20 \%$. The measured data are shown in Section III. Contour plots of the capacitance density across $H$ vs. $S$ where it is assumed that $W=T=S$ are also shown.

\section{Capacitor Model}

2.1. Fabrication Steps

Figure 2 illustrates fabrication steps for the chip structure shown in Figure 1b. CMOS is fabricated for word-line (WL) and bit-line (BL) decoders in memory array and control circuits in the periphery at step (a). The first level WL is patterned above the decoding transistors at step (b). The same pattern remains above the control circuits in the periphery, which will be used as terminals of scalable capacitors. The first-level memory elements are formed in the memory array at step (c). The material for the memory elements is removed out of the periphery. Dielectric film is placed across a wafer to create isolation between the next-neighbor memory elements in the memory array and between the first and second metal wires in the periphery. The second level wires are patterned for the first-level BL in the memory array and for scalable capacitors in the periphery at step (d). The second-level memory elements are formed in the memory array and they are removed out of the periphery at step (e). One can repeat steps (b) through (e) to have multiple levels of WLs and BLs in the memory array and a denser capacitor structure in the periphery. After the memory array structure is finalized, additional metal wires are placed to connect the WLs and BLs with the decoding transistors in the memory array and to connect the wires in scalable capacitors with circuits in the periphery at step (f).

(a)

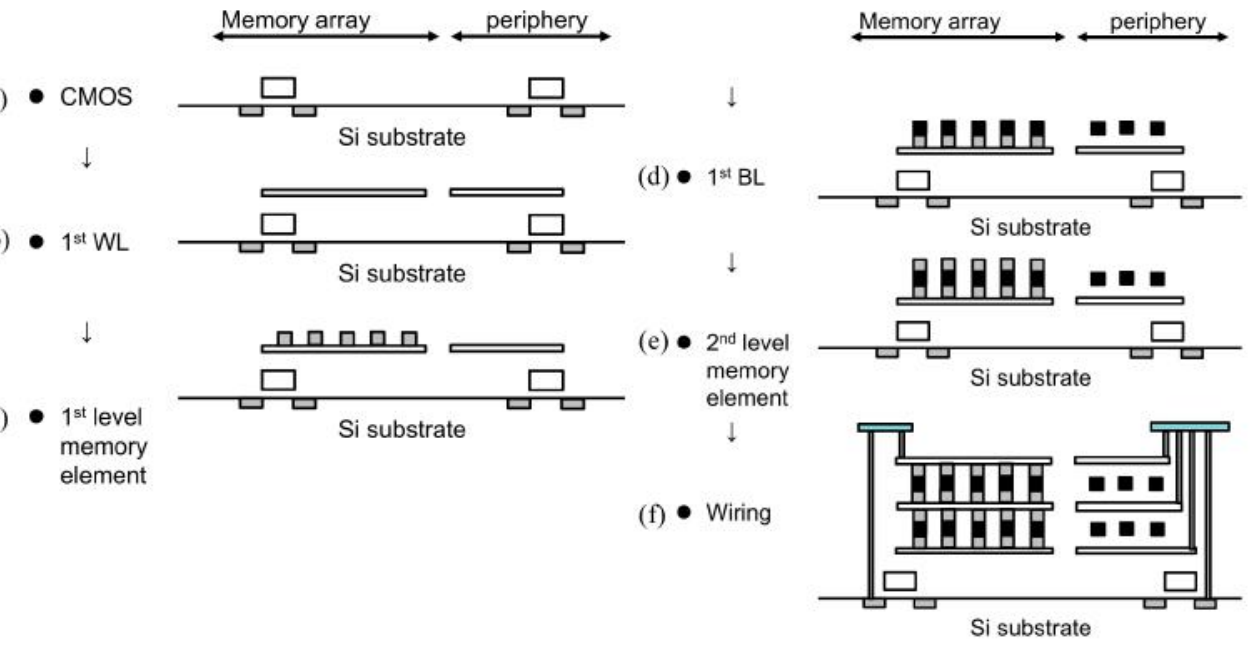

Figure 2. Fabrication steps from CMOS (a) through wiring $(\mathbf{b}-\mathbf{f})$.

\subsection{Three-Capacitor Structures Studied in This Paper}

To investigate which structure is the best in terms of capacitance density, i.e., capacitance per area as a function of process parameters such as the height $(H)$ and thickness $(T)$ of wires and design rules such as the width $(W)$ and space $(S)$ of wires, three capacitor structures are modeled. Figure 3 shows the three capacitor structures: vertical capacitor $C_{V}$ (a) and vertical and horizontal capacitors $C_{V H}$ (b) and $C_{V H 2}$ (c). Even and odd numbers of wires are connected to the first and second terminals in $C_{V H}$, whereas two next-neighbor wires are connected to the first and second terminals in $C_{V H 2}$, alternately. It is assumed that every level of wires has the same values for $H, T, W$, and $S$. 


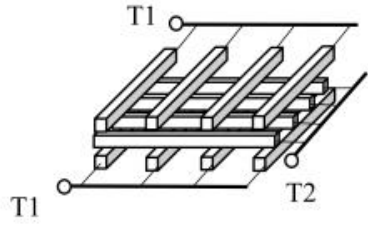

(a)

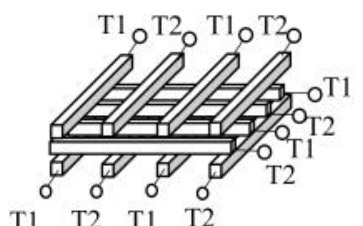

(b)

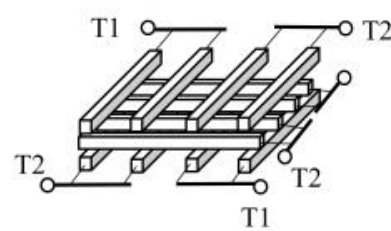

(c)

Figure 3. Three capacitor structures studied in this paper: vertical capacitor $C_{V}(\mathbf{a})$, and vertical and horizontal capacitors $C_{V H}(\mathbf{b})$ and $C_{V H 2}(\mathbf{c})$.

\subsection{Modeling}

To estimate the capacitance of each structure of Figure 3, Wong's model [18] is utilized. The vertical capacitance component $C_{V E R}$ as shown in Figure $4 \mathrm{a}$ is composed of $C_{1}, C_{2}$, and $C_{3}$ as shown in Figure $4 \mathrm{~b}$. According to [18], each of the capacitance components are given by (2), (3), and (4), respectively.

$$
\begin{aligned}
& C_{V E R}=C_{1}+C_{2}+C_{3} \\
& \frac{C_{1}}{\varepsilon}=\frac{W^{2}}{H} \\
& \frac{C_{2}}{\varepsilon}=3.73 W^{0.6} S^{0.4} \times\left(\frac{T}{T+0.035 H}\right)^{0.64} \times\left(\frac{T}{T+0.851 S}\right)^{0.12} \times \frac{H}{H+0.051 S} \times \exp \left(\frac{-H}{0.7(S+0.4 H)}\right) \\
& \frac{C_{3}}{\varepsilon}=3.73 W^{0.6} S^{0.3} \times\left(\frac{T}{T+0.035 H}\right)^{0.64} \times \exp \left(\frac{-H}{0.7(S+0.4 H)}\right) \times\left(\frac{H}{H+0.015 S}\right)^{3}
\end{aligned}
$$

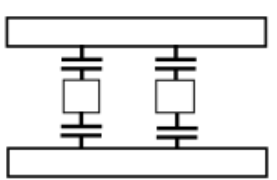

\section{$\square \mathrm{H} \square$}

(a)

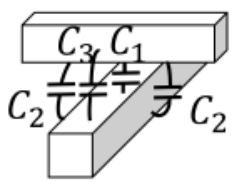

(b)

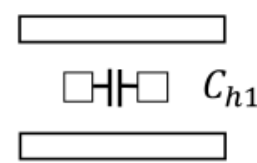

(d) (c)

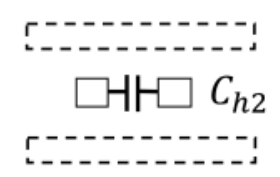

(e)

Figure 4. Breakdown of the capacitor; vertical capacitance components $(\mathbf{a}, \mathbf{b})$ and horizontal capacitance components (c-e).

The horizontal capacitance component $C_{H O R}$ as shown in Figure $4 \mathrm{c}$ is given by either $C_{h 1}$ or $C_{h 2}$, as shown in Figure $4 \mathrm{~d}$ or Figure $4 \mathrm{e}$, respectively. $C_{h 1}$ is the horizontal capacitance that sees adjacent levels of wires above and below, whereas $C_{h 2}$ is the horizontal capacitance that does not see adjacent levels of wires above and below. Based on [18], each of the capacitance components are given by (5) and (6), respectively.

$$
\begin{aligned}
\frac{C_{h 1}}{\varepsilon}= & 1.144 \frac{T}{S}\left(\frac{2 H+T}{2 H+T+2.059 S}\right)^{0.0944}+0.7428\left(\frac{W}{W+1.592 S}\right)^{1.144} \\
& +1.158\left(\frac{W}{W+1.874 S}\right)^{0.1612} \times\left(\frac{2 H+T}{2 H+T+0.9801 S}\right)^{1.179}
\end{aligned}
$$




$$
\begin{aligned}
\frac{C_{h 2}}{\varepsilon}= & 1.412 \frac{T}{S} \exp \left(-\frac{2 S}{S+8.014 H}-\frac{2 S}{S+8.014 H}\right)+1.1852\left(\frac{W}{W+0.3078 S}\right)^{0.25724} \\
& \times\left\{\left(\frac{H}{H+8.961 S}\right)^{0.7571}+\left(\frac{H}{H+8.961 S}\right)^{0.7571}\right\} \times \exp \left(-\frac{2 S}{S+6 H}\right)
\end{aligned}
$$

For simplicity, this paper discusses the capacitors whose shape is square with a length of $L$. Therefore, the number of wires in each level $(N)$ is $L /(W+S)$. When the number of levels of wires is $M$, the number of each capacitance component is given by Table 1 .

Table 1. Number of capacitance components for the three capacitor structures.

\begin{tabular}{ccc}
\hline & C VER (Figure 4a) & C HOR $_{\text {(Figure 4c) }}$ \\
\hline$C_{V}$ (Figure 3a) & $(M-1) N^{2}$ & None \\
$C_{V H}$ (Figure 3b) & $\left(M-1 N^{2} / 2\right.$ & $M\left(C_{h 1} N(N-1)+C_{h 2}(N-1)^{2}\right)$ \\
$C_{V H 2}$ (Figure 3c) & $\left(M-1 N^{2} / 2\right.$ & $M\left(C_{h 1} N / 2(N-1)+C_{h 2}(N / 2-1)(N-1)\right)$ \\
\hline
\end{tabular}

As a result, $C_{V}, C_{V H}$, and $C_{V H 2}$ are given by (7), (8), and (9), respectively.

$$
\begin{gathered}
C_{V}=(M-1) N^{2} C_{V E R} \\
C_{V H}=(M-1) N^{2} / 2 C_{V E R}+M\left(C_{h 1} N(N-1)+C_{h 2}(N-1)^{2}\right) \\
C_{V H 2}=(M-1) N^{2} / 2 C_{V E R}+M\left(C_{h 1} N / 2(N-1)+C_{h 2}(N / 2-1)(N-1)\right)
\end{gathered}
$$

\subsection{Comparison of the Capacitance Density between the Three Structures}

In this section, various projections of the capacitance density based on the model given by (1) through (9) are studied. It is assumed that $S=W$ to minimize the bit cost. The aspect ratio of $T / W$ can be more arbitrary, e.g., 1, 1.5 or 2 as a scaling rule, but in this study, the aspect ratio is assumed to be one. $H$ is varied regardless of $S, W$, and T. Since Wong's model has a good accuracy when the ratio of the maximum value among $W, S, T$, and $H$ to the minimum value among them is between 1 and 8 [15], those four parameters are varied from 5 to $40 \mathrm{~nm}$ in this study.

Figure 5a shows the capacitance of the vertical capacitor $\mathrm{C}_{\mathrm{V}}$ shown in Figure $3 \mathrm{a}$ as a function of $H$ when $M=3$. The "parallel plate model" indicates that the capacitance is modeled by parallel plates where $C=\varepsilon / H$ as if three levels of wires are treated as plates rather than wires. When $C_{V}$ is approximated by a form of $C_{V} \sim H^{\alpha}$, the power $\alpha$ is about -0.90 and -0.60 for $W=S=T=5 \mathrm{~nm}$ and $40 \mathrm{~nm}$, respectively. It seems that the parallel plate model can predict the capacitance well for $W=S=T=H$, but the discrepancy increases when the difference between $W(=S=T)$ and $H$ increases. Figure $5 \mathrm{~b}$ shows the capacitance of the vertical/horizontal capacitor $C_{V H}$ shown in Figure $3 \mathrm{~b}$ as a function of $W(=S=T)$. The "parallel plate model" indicates that the capacitance is modeled by parallel plates where $C=\varepsilon / 4(2 M / S+(M-1) / H)$. The first term is originated from the capacitance between next neighbors such as $C_{h 1}$ and $C_{h 2}$ of Figure 5 , and the second term is from the crossover capacitance as $C_{1}$ of Figure 5 .

To see which capacitor structure has the highest capacitance across the $S-H$ plane, the capacitance ratios of $C_{V} / C_{V H}$ and $C_{V H 1} / C_{V H}$ in case of $\mathrm{M}$ of 3 are shown in Figure 6a,b, respectively. There is a boundary between $C_{V}$ and $C_{V H}$ in Figure 6a; the more scaled in $S$, the larger $C_{V H}$, and the more scaled in $H$, the larger $C_{V}$. On the other hand, there is no boundary between $C_{V H}$ and $C_{V H 2}$ in Figure $6 \mathrm{~b}$. As a result, the capacitor structure with the highest capacitance density can be either $C_{V}$ or $C_{V H}$ depending on the parameter condition on $S$ and $H$. 


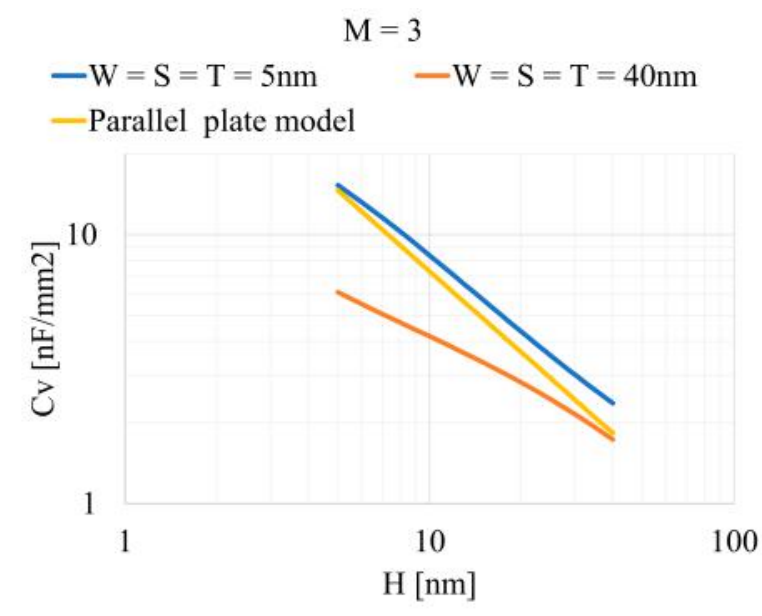

(a)

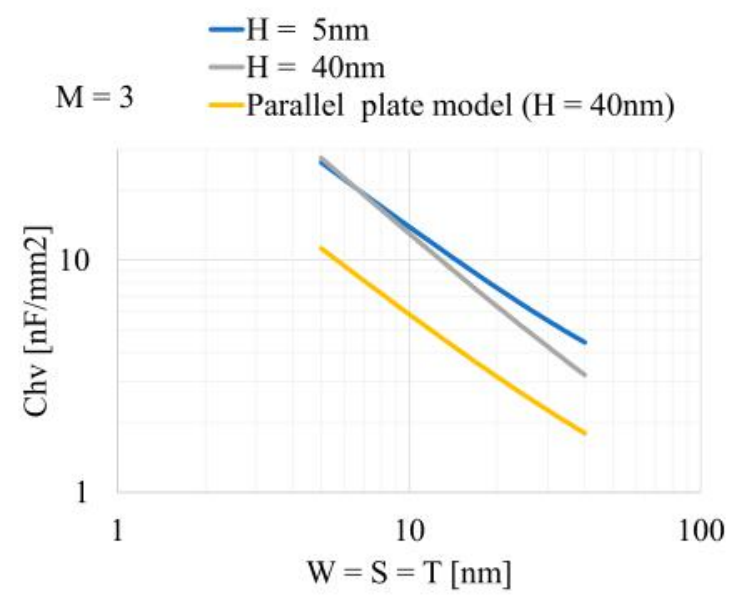

(b)

Figure 5. (a) $H$ vs. $C_{V}$ and (b) $W(=S=T)$ vs. $C_{\mathrm{HV}}$ in case the number of wires is three.

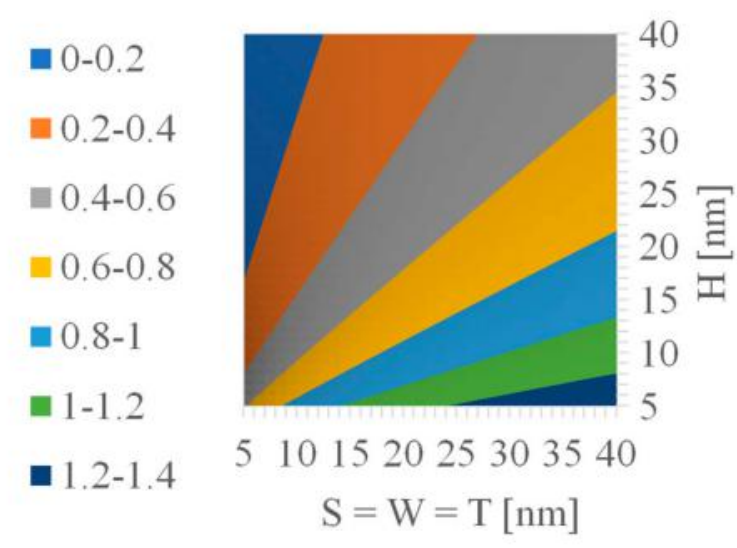

(a)

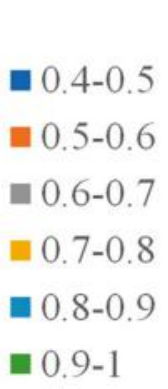
510152025303540 $\mathrm{S}=\mathrm{W}=\mathrm{T}[\mathrm{nm}]$

Figure 6. (a) $C_{V} / C_{V H}$ and (b) $C_{V H 1} / C_{V H}$ in case the number of wires is three.

The boundary condition depends on the number of metal wires, as shown in Figure 7. As the number of metal wires increases, $C_{V E R}$ has more impact on the total capacitance than $C_{H O R}$ because $C_{V E R} / C_{H O R}$ is proportional to $1-1 / M$. As a result, the boundary shifts toward the left top with a larger slope, as shown in Figure 7.

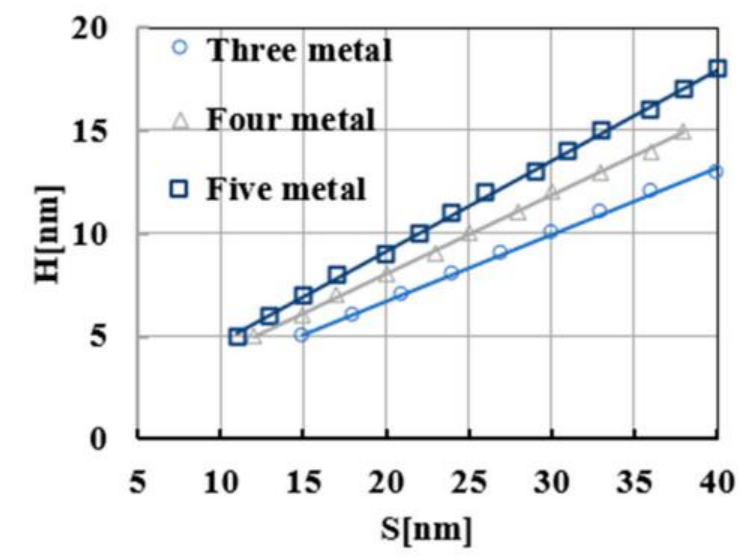

Figure 7. Boundary conditions on the $S-H$ plane for the memory with three, four, and five metal wires.

When the parameters are somewhere in the area below the boundary line of Figure 8 , the $C_{V}$ structure should be used for higher capacitance density; otherwise, the $C_{V H}$ structure 
should be used. One can determine the best structure and the value of the capacitance density based on such a figure as Figure 8 easily once the process and design rules have been determined.
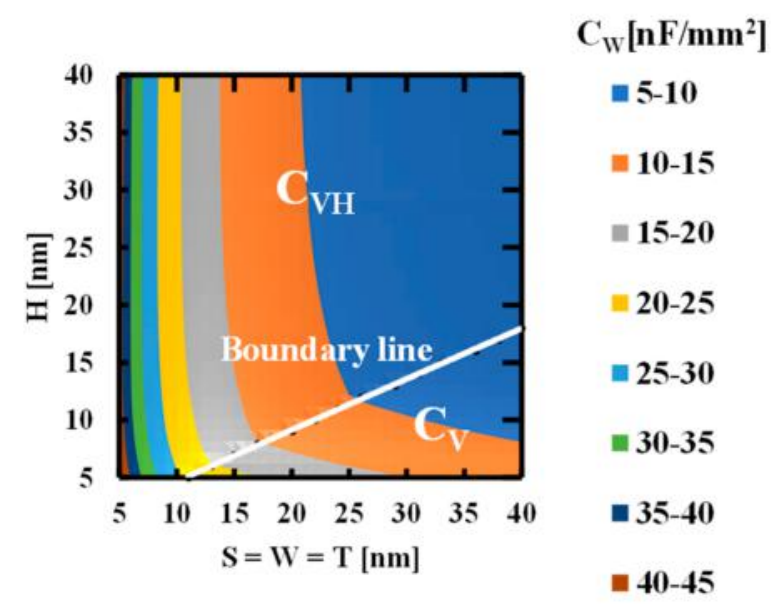

Figure 8. Contour plot of wring capacitance across the $H-S$ plane in case of $M=5$.

\subsection{Scalability of Capacitance across 3D Crosspoint Memory Technology}

Figure 9 shows increases in capacitance across 3D crosspoint memory technology. $C_{V H}$ is the best structure for $S=W=T=10 \mathrm{~nm}$ and $H=20 \mathrm{~nm}$, as shown in Figure 9a, whereas $C_{V}$ is the best one for $S=W=T=10 \mathrm{~nm}$ and $H=20 \mathrm{~nm}$, as shown in Figure $9 \mathrm{~b}$. One can expect the increase in capacitance density by $8.2 \mathrm{nF} / \mathrm{mm}^{2}$ or $7.1 \mathrm{nF} / \mathrm{mm}^{2}$ per increase in the number of layers of two, respectively.

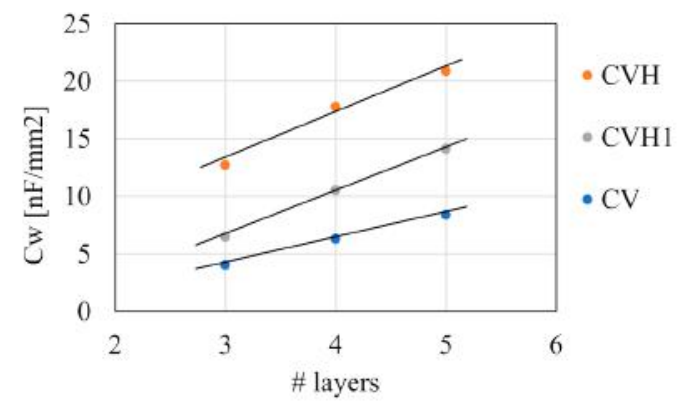

(a)

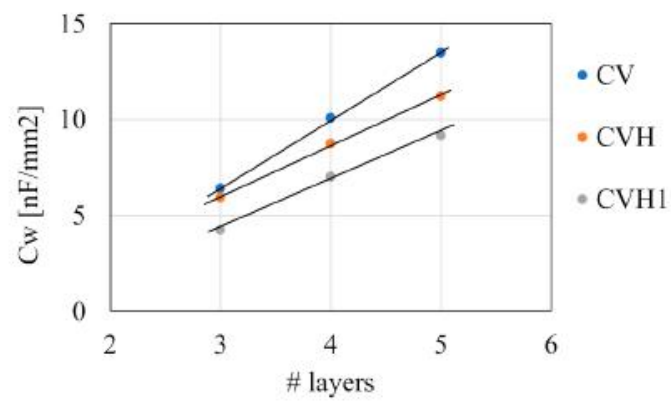

(b)

Figure 9. Capacitance vs. the number of wring layers: (a) $S=W=T=10 \mathrm{~nm}, H=20 \mathrm{~nm}$, (b) $S=W=T=25 \mathrm{~nm}, H=7 \mathrm{~nm}$.

\section{Measurement}

To validate the model, twelve types of capacitor structure as shown in Table 2 were fabricated in $180 \mathrm{~nm} 6$ metal CMOS, as shown in Figure 10. Each structure has $500 \mu \mathrm{m} \times 300 \mu \mathrm{m}$. Note that the $H$ and $T$ parameters are not allowed to be disclosed.

Table 2. Fabricated test structures for scalable capacitors.

\begin{tabular}{|c|c|c|c|c|c|c|c|c|c|c|c|c|}
\hline & 1 & 2 & 3 & 4 & 5 & 6 & 7 & 8 & 9 & 10 & 11 & 12 \\
\hline$S=W[\mu \mathrm{m}]$ & \multicolumn{6}{|c|}{$0.28 \mu \mathrm{m}$} & \multicolumn{6}{|c|}{$3.0 \mu \mathrm{m}$} \\
\hline \# wires & & 3 & & & 4 & & & 3 & & & 4 & \\
\hline$C$ structure & $C_{V}$ & $C_{V H}$ & $C_{V H 2}$ & $C_{V}$ & $C_{V H}$ & $C_{V H 2}$ & $C_{V}$ & $C_{V H}$ & $C_{V H 2}$ & $C_{V}$ & $C_{V H}$ & $C_{V H 2}$ \\
\hline
\end{tabular}




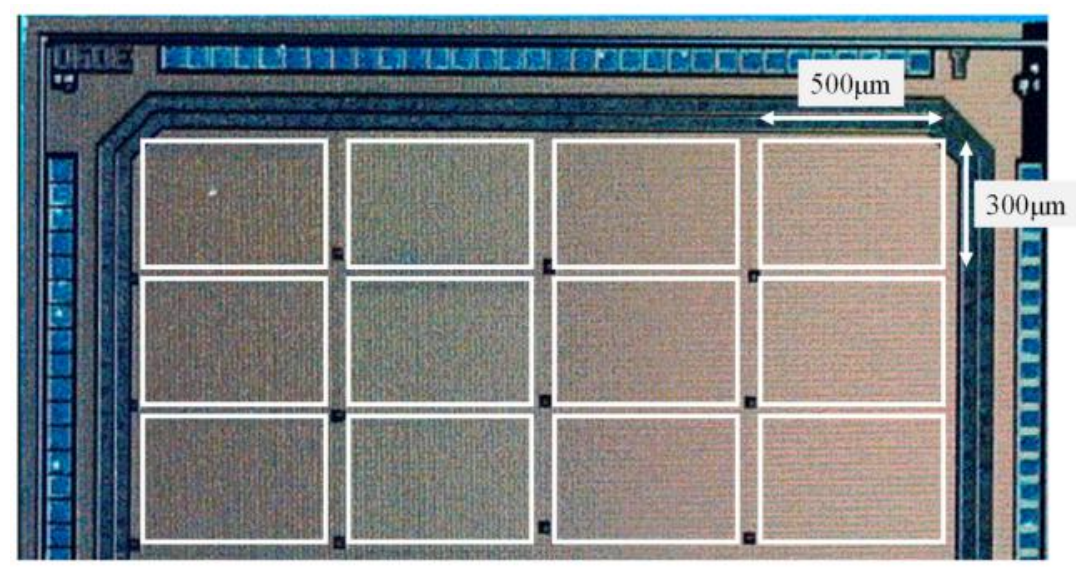

Figure 10. Die photo.

The capacitance of each capacitor is measured and is subtracted by that of no structure, which only has pad and wiring capacitance to connect to a measurement tool. Two dies were measured and compared with the model, as shown in Figure 11. The errors between the measured data and the calculated one using the model were $20 \%$ at the largest. As projected by the model, $\mathrm{C}_{\mathrm{VH}}$ is the best capacitor structure when the wires are as narrow as $0.28 \mu \mathrm{m}$ and as short space as $0.28 \mu \mathrm{m}$, while $C_{V}$ is the best capacitor structure when the wires are as wide as $3.0 \mu \mathrm{m}$ and as large space as $3.0 \mu \mathrm{m}$. Even though the number of capacitor types was limited, the model was validated.

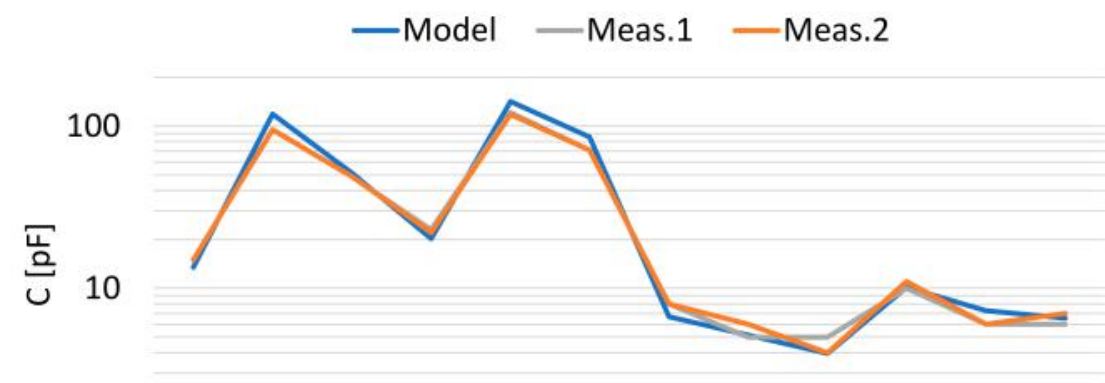

\begin{tabular}{|c|c|c|c|c|c|c|c|c|c|c|c|c|}
\hline$=\mathrm{W}$ & 0.28 & 0.28 & 0.28 & 0.28 & 0.28 & 0.28 & 3.0 & 3.0 & 3.0 & 3.0 & 3.0 & 3.0 \\
\hline \#layers & 3 & 3 & 3 & 4 & 4 & 4 & 3 & 3 & 3 & 4 & 4 & 4 \\
\hline & 1 & 2 & 3 & 1 & 2 & 3 & 1 & 2 & 3 & 1 & 2 & 3 \\
\hline
\end{tabular}

Figure 11. Measured vs. model capacitance.

\section{Discussions}

Table 3 summarizes the differences from the previous studies. In [18], it is assumed that each level of wires is routed perpendicularly to lower and upper levels, and there is no via placed on the wires. Thus, three-dimensional crossover capacitance was modeled. In [19], vias are included to increase the capacitance per area, and vertical parallel plate and vertical bars are considered as well as a horizontal parallel plate. On the other hand, in this work, wires are routed as in [18] with no vias because the design rule of the capacitor is the same as the memory array where the pitch is tighter than the design rule allowing vias, but other capacitor structures such as $C_{V}$ and $C_{V H 2}$ are discussed as well as $C_{V H}$. 
Table 3. Comparison with the previous studies $[18,19]$.

\begin{tabular}{cccc}
\hline & Wong [18] & Aparico [19] & This Work \\
\hline Studied structure & $C_{V H}$ & Horizontal and vertical parallel & $C_{V}, C_{V H}, C_{V H 2}$ \\
With vias? & No & plate, Vertical bars & Yes \\
\hline
\end{tabular}

In this work, only the capacitance density is considered. To design the capacitor array, one needs to determine the size of the unit capacitor. Since the wires of the capacitor need to be connected at the edges of the array due to mismatch in the design rules between the inside and outside of the memory array, the operation frequency of the capacitors decreases as the capacitor array size increases. Therefore, how to determine the capacitor unit size needs to be given for circuit designers as a function of the required operation frequency, which needs to be done in the future work. When the capacitors are used in DACs and comparators, the matching property of the capacitors can limit the accuracy of such analog circuits. The matching property also needs to be addressed in the future work.

\section{Conclusions}

This paper studied the design of scalable capacitors for 3D crosspoint memory. Three types of structures namely $C_{V}, C_{V H}$, and $C_{V H 2}$ were modeled, and their capacitance density was compared across $S(=W=T)$ and $H$. The boundary conditions to determine the best capacitor structure were shown under the condition of $W=S=T$ for the memory technology with three, four, and five levels of wires as demonstration. The test structures were fabricated in $180 \mathrm{~nm}$ standard CMOS to validate the models. Since the capacitance model includes $W, S, T$, and $H$ as independent parameters, one can identify the best structure based on the models once the technology including the number of wires and process parameters such as $H$ and $T$, and the design rules of $S$ and $W$ are determined. In this paper, only the capacitance density was focused. Therefore, how to determine the capacitor array size needs to be given for circuit designers as a function of the required operation frequency, which should be done in the future work.

Author Contributions: Conceptualization, T.T.; methodology, Y.T. and T.T.; software, Y.T.; validation, Y.T. and T.T.; formal analysis, Y.T. and T.T.; investigation, Y.T. and T.T.; writing-original draft preparation, Y.T.; writing-review and editing, T.T.; funding acquisition, T.T. All authors have read and agreed to the published version of the manuscript.

Funding: This research received no external funding.

Acknowledgments: This work is supported by Kioxia Corp., d-lab. VDEC, Synopsys, Inc., Cadence Design Systems, Inc. Rohm Corp.

Conflicts of Interest: The authors declare no conflict of interest.

\section{Nomenclature}

W Width of each wire

$S \quad$ Space between wires

$T \quad$ Thickness of wires

$H \quad$ Height of dielectric film between levels of wires

$C_{V E R} \quad$ Vertical capacitance component

$C_{H O R}$ Horizontal capacitance component

$C_{V} \quad$ Vertical capacitor

$C_{V H} \quad$ Vertical and horizontal capacitors where even and odd numbers of wires are connected to the first and second terminals

$C_{V H 2}$ Vertical and horizontal capacitors where two next neighbor wires are connected to the first and second terminals 


\section{References}

1. Kau, D.C.; Tang, S.; Karpov, I.V.; Dodge, R.; Klehn, B.; Kalb, J.A.; Strand, J.; Diaz, A.; Leung, N.; Wu, J.; et al. A stackable cross point phase change memory. In Proceedings of the 2009 IEEE International Electron Devices Meeting (IEDM), Baltimore, MD, USA, 7 December 2009; pp. 1-4. [CrossRef]

2. Kim, T.; Choi, H.; Kim, M.; Yi, J.; Kim, D.; Cho, S.; Lee, H.; Hwang, C.; Hwang, E.R.; Song, J.; et al. High-performance, cost-effective $2 \mathrm{z}$ nm two-deck crosspoint memory integrated by self-align scheme for $128 \mathrm{~Gb}$ SCM. In Proceedings of the 2018 IEEE International Electron Devices Meeting (IEDM), San Francisco, CA, USA, 1-5 December 2018; pp. 37.1.1-37.1.4. [CrossRef]

3. Chien, W.C.; Ho, H.Y.; Yeh, C.W.; Yang, C.H.; Cheng, H.Y.; Kim, W.; Kuo, I.T.; Gignac, L.M.; Lai, E.K.; Gong, N.; et al. Comprehensive scaling study on 3D cross-point PCM toward 1Znm node for SCM applications. In Proceedings of the 2019 Symposium on VLSI Technology, Kyoto, Japan, 9-14 June 2019; pp. 60-61. [CrossRef]

4. Chevallier, C.J.; Siau, C.H.; Lim, S.F. A $0.13 \mu \mathrm{m} 64 \mathrm{Mb}$ multi-layered conductive metal-oxide memory. In Proceedings of the 2010 IEEE International Solid-State Circuits Conference (ISSCC), San Francisco, CA, USA, 7 February 2010; pp. 260-261. [CrossRef]

5. Kawahara, A.; Azuma, R.; Ikeda, Y.; Kawai, K.; Katoh, Y.; Hayakawa, Y.; Tsuji, K.; Yoneda, S.; Himeno, A.; Shimakawa, K.; et al. An $8 \mathrm{Mb}$ Multi-Layered Cross-Point ReRAM Macro With $443 \mathrm{MB} / \mathrm{s}$ Write Throughput. IEEE J. Solid-State Circuits 2013, 48, 178-185. [CrossRef]

6. Yu, S.; Deng, Y.; Gao, B.; Huang, P.; Chen, B.; Liu, X.; Kang, J.; Chen, H.Y.; Jiang, Z.; Wong, H.S. Design Guidelines for 3D RRAM Cross-Point Architecture. In Proceedings of the IEEE International Symposium on Circuits and Systems (ISCAS), Melbourne, Australia, 1 June 2014; pp. 421-424. [CrossRef]

7. Zhou, J.; Kim, K.H.; Lu, W. Crossbar RRAM Arrays: Selector Device Requirements during Read Operation. IEEE Trans. Electron Devices 2014, 61, 1369-1376. [CrossRef]

8. Chiu, P.-F.; Nikoli, B. A differential 2R crosspoint RRAM array with zero standby current. IEEE Trans. Circuits Syst. II Exp. Briefs 2015, 62, 461-465. [CrossRef]

9. Andersen, T.M.; Krismer, F.; Kolar, J.W.; Toifl, T.; Menolfi, C.; Kull, L.; Morf, T.; Kossel, M.; Brändli, M.; Buchmann, P.; et al. A sub-ns response on-chip switched-capacitor DC-DC voltage regulator delivering $3.7 \mathrm{~W} / \mathrm{mm} 2$ at $90 \%$ efficiency using deep-trench capacitors in 32nm SOI CMOS. In Proceedings of the IEEE International Solid-State Circuits Conference Digest of Technical Papers (ISSCC), San Francisco, CA, USA, 9-13 February 2014. [CrossRef]

10. Pei, C.; Booth, R.; Ho, H.; Kusaba, N.; Li, X.; Brodsky, M.; Parries, P.; Shang, H.; Divakaruni, R.; Iyer, S. A novel, low-cost deep trench decoupling capacitor for high-performance, low-power bulk CMOS applications. In Proceedings of the International Conference on Solid-State and Integrated-Circuit Technology, Beijing, China, 20-23 October 2008; pp. 1146-1149. [CrossRef]

11. El-Damak, D.; Bandyopadhyay, S.; Chandrakasan, A.P. A 93\% Efficiency Reconfigurable Switched-Capacitor DC-DC Converter Using On-Chip Ferroelectric Capacitors. In Proceedings of the IEEE International Solid-State Circuits Conference Digest of Technical Papers, San Francisco, CA, USA, 17-21 February 2013; pp. 374-375. [CrossRef]

12. Tanzawa, T.; Murakoshi, T.; Kamijo, T.; Tanaka, T.; McNeil, J.J.; Duesman, K. Design challenge in 3D NAND technology: A 4.8X area- and 1.3X power-efficient 20V charge pump using tier capacitors. In Proceedings of the IEEE Asian Solid-State Circuits Conference, Toyama, Japan, 7-9 November 2016; pp. 165-168. [CrossRef]

13. Somasekhar, D.; Srinivasan, B.; Pandya, G.; Hamzaoglu, F.; Khellah, M.; Karnik, T.; Zhang, K. Multi-phase 1GHz voltage doubler charge-pump in 32nm logic process. IEEE J. Solid-State Circuits 2010, 45, 751-758. [CrossRef]

14. Tanzawa, T. On-chip switched-capacitor DC-DC converter in memory technology: State of the art and challenges. In Proceedings of the European Conference on Circuit Theory and Design (ECCTD), Catania, Italy, 4-6 September 2017. [CrossRef]

15. Ruehli, A.; Brennan, P.A. Capacitance models for integrated circuit metallization wires. IEEE J. Solid-State Circuits 1975, 10, 530-536. [CrossRef]

16. Arora, N.D.; Raol, K.V.; Schumann, R.; Richardson, L.M. Modeling and extraction of interconnect capacitances for multilayer VLSI circuits. IEEE Trans. Comput.-Aided Des. Integr. Circuits Syst. 1996, 15, 58-67. [CrossRef]

17. Wong, S.C.; Liu, P.S.; Ru, J.W.; Lin, S.T. Interconnection capacitance models for VLSI circuits. Solid-State Electron. 1998, 42, 969-977. [CrossRef]

18. Wong, S.C.; Lee, T.G.Y.; Ma, D.J.; Chao, C.J. An Empirical Three-Dimensional Crossover Capacitance Model for Multilevel Interconnect VLSI Circuits. IEEE Trans. Semicond. Manuf. 2000, 13, 219-227. [CrossRef]

19. Aparicio, R.; Hajimiri, A. Capacity limits and matching properties of integrated capacitors. IEEE J. Solid-State Circuits 2002, 37, 384-393. [CrossRef] 